\title{
Reflets
}

Revue ontaroise d'intervention sociale et communautaire

\section{L'usage abusif de substances toxiques par les femmes violentées : les effets d'une victimisation sociale}

\section{Francine Rivette}

Volume 6, numéro 1, printemps 2000

Approches d'intervention : définir et renouveler nos pratiques

URI : https://id.erudit.org/iderudit/026308ar

DOI : https://doi.org/10.7202/026308ar

Aller au sommaire du numéro

Éditeur(s)

Reflets : Revue ontaroise d'intervention sociale et communautaire

ISSN

1203-4576 (imprimé)

1712-8498 (numérique)

Découvrir la revue

Citer cet article

Rivette, F. (2000). L'usage abusif de substances toxiques par les femmes violentées : les effets d'une victimisation sociale. Reflets, 6(1), 232-249.

https://doi.org/10.7202/026308ar

Tous droits réservés (C) Reflets : Revue ontaroise d'intervention sociale et communautaire, 2000
Ce document est protégé par la loi sur le droit d'auteur. L'utilisation des services d'Érudit (y compris la reproduction) est assujettie à sa politique d'utilisation que vous pouvez consulter en ligne.

https://apropos.erudit.org/fr/usagers/politique-dutilisation/ 


\section{L'usage abusif de substances toxiques par les femmes violentées : les effets d'une victimisation sociale}

Francine Rivette

Récipiendaire de la bourse RIFAS 1999, École de service social, Université d'Ottawa ${ }^{1}$

La violence faite aux femmes fait partie de l'histoire de l'humanité. Si la violence demeure un phénomène régulier, plus facile à reconnaitre dans les comportements agressifs et les paroles blessantes, elle exprime aussi des rapports de force plus subtils. Comme elle se présente sous plusieurs formes, il est parfois difficile de la définir. Par contre, ce qui est clair, c'est qu'elle impose des limites et maintient des rapports de domination. Et ce sont les femmes qui en souffrent le plus, particulièrement dans le cadre de relations conjugales. L'abus de substances toxiques est une réalité qui influence aussi la vie des femmes violentées. L'exploration des différentes facettes de la toxicomanie chez ces femmes permet de mieux comprendre la relation entre les deux phénomènes.

La démarche exploratoire, dont il est question dans cet article, a pour objectif de répondre au besoin de connaissances sur l'aide recherchée par les femmes ayant vécu de la violence conjugale. Celles-ci vivent des expériences traumatisantes et ont parfois recours aux substances toxiques pour gérer leurs émotions. Les problèmes de toxicomanie qu'elles présentent peuvent devenir un obstacle additionnel qui freine leur recherche d'aide et le processus de changement. Elles ont besoin d'une aide concrète 
pour se réapproprier un pouvoir et affronter leur situation. Afin d'identifier les formes d'aide dispensées pour répondre aux besoins des femmes violentées, nous présentons cette étude exploratoire réalisée auprès de sept organismes des trois comtés unis de Stormont, Dundas et Glengarry dans le Sud-est ontarien, qui offrent des services pour les femmes ayant vécu la violence conjugale et pour celles abusant de substances toxiques.

\section{La recherche d'aide des femmes violentées}

Une recension des écrits portant sur les stratégies adoptées par les femmes pour mettre fin à la violence subie (Prairie 1984) associe deux aspects importants, soit les éléments déclencheurs de la rupture (Galley et Werk 1994; Cantin et Rinfret-Raynor 1994) et les processus de recherche d'aide (Bilodeau 1987 et 1994; Dobash et Dobash 1985; Limandri 1985).

Au niveau des éléments déclencheurs de la rupture, toute démarche est particulière à chaque femme, mais on peut aussi repérer plusieurs facteurs communs, dont le niveau de motivation lié au désir de changement, le degré d'ouverture pour discuter de l'expérience (Limandri 1985) et, enfin, le but poursuivi qui évolue de la tolérance à la violence vers le refus de la violence pour en arriver à la cessation de la violence (Bilodeau 1987). Ainsi, certaines auteures établissent un lien entre la décision de quitter la relation abusive et l'attribution de la responsabilité de la violence (Galley et Werk 1994). D'autres comparent et établissent des différences entre la situation des femmes violentées vivant avec leur conjoint et celles qui sont séparées du conjoint violent (Cantin et RinfretRaynor 1994). En général, les résultats des études démontrent que la décision de quitter un conjoint violent est en constante évolution (Cantin et Rinfret-Raynor 1994), mais que ce processus est influencé par la recherche d'aide dans l'environnement (Bilodeau 1987). En retour, la réponse de l'environnement influence aussi la recherche d'aide des femmes et leurs réactions face à la violence subie. C'est la raison pour laquelle nous avons 
choisi d'examiner l'influence des services sur la recherche d'aide des femmes dans le cadre de la présente étude.

\section{Abus de substances et violence faite aux femmes}

Afin de répondre efficacement aux besoins des femmes violentées lors de demande d'aide, une évaluation s'avère essentielle afin de présenter des choix et des solutions qui correspondent à leur réalité. Puisque l'abus de substances toxiques peut influencer la vie des femmes violentées, il importe donc de cerner la relation entre la violence faite aux femmes et l'usage abusif de substances toxiques, sans nécessairement établir de lien de causalité entre les deux phénomènes.

Selon les études canadiennes les plus récentes portant sur la consommation de drogues, l'usage abusif de substances toxiques est plus élevé chez les personnes qui vivent ou qui ont vécu de la violence que dans l'ensemble de la population (Statistique Canada 1994; Ratner 1993; Groeneveld et Shain 1989). Les résultats présentés par Statistique Canada, en 1994, révèlent que les femmes canadiennes invoquent la violence qu'elles subissent ou ont subi comme motifs à leur consommation de substances toxiques chez, respectivement, $12 \%$ des femmes consommant de l'alcool, $9 \%$ consommant des drogues ou des médicaments et enfin, $5 \%$ consommant de l'alcool, des drogues et des médicaments. Ainsi, la consommation de substances toxiques devient pour plusieurs femmes violentées, un moyen de survie.

Certaines études portant sur la toxicomanie féminine démontrent une relation directe entre la problématique de la violence conjugale et l'abus de substances toxiques (Fondation de la recherche sur la toxicomanie (FRT) 1995; Statistique Canada 1994; Ratner 1993; Groeneveld et Shain 1989). Ainsi, une étude révèle une consommation abusive de substances psychoanaleptiques parmi les femmes violentées (Groeneveld et Shain 1989), alors qu'une autre découvre une dépendance à l'alcool plus élevée parmi les femmes ayant vécu la violence 
physique (Ratner 1993). Toutefois, certains auteurs considèrent que les femmes en général ont moins tendance que les hommes à boire de l'alcool pour gérer leurs conflits (Adlaf 1993; Biener 1987). L'étude de Groeneveld et Shain (1989), réalisée auprès de 2100 femmes adultes et portant sur la consommation abusive de substances psychoanaleptiques, précise que les femmes agressées sexuellement à l'âge adulte consomment plus de médicaments sur ordonnance ou en vente libre et que les femmes ayant vécu la violence physique dans les relations intimes ont davantage tendance à utiliser les somniferes et les calmants.

\section{Le langage de l'usage abusif}

Pour dépister et cerner la problématique de dépendance des femmes violentées et comprendre les effets d'une drogue, on doit tenir compte de plusieurs facteurs tels que la quantité de la drogue consommée, le mode d'administration, le type de drogues consommées et l'interaction des diverses drogues consommées simultanément (FRT 1995). Par rapport aux drogues illicites, la variété des préparations rend impossible de tenir compte uniquement de la quantité consommée ou de la qualité du produit, ce qui complique d'autant l'évaluation ou le contrôle des effets. Il en découle qu'une femme, consommant plusieurs substances à la fois ou consommant des substances «de rue», ne peut pas prévoir les effets qu'auront ces substances, ce qui contribue à la maintenir en position d'impuissance.

La consommation comporte plusieurs modes de gestion pour les femmes. En effet, la polytoxicomanie, comme la consommation simultanée d'alcool, de cocaïne et de tranquillisants, modifie notre compréhension de la dépendance aux substances qui revêt alors une forme diversifiée et changeante (Landry et al. 1994 : 182). C'est pourquoi il importe de définir certaines notions clés propres à l'abus de substances afin de cerner davantage l'expérience vécue par les femmes. 
Tremblay définit le psychotrope comme étant «toute drogue dont une des actions principales s'exerce sur le système nerveux central, entraînant des modifications du psychisme et du comportement» (1988 :241). Pour cet auteur, la tolérance à une drogue se manifeste lorsqu'une personne consomme régulièrement cette drogue, ayant besoin d'une quantité toujours plus grande pour ressentir les mêmes effets. Tremblay (1988) décrit le développement de la tolérance comme étant «un phénomène physiologique, résultant de l'adaptation de l'organisme à absorber et à métaboliser un produit consommé régulièrement». Par ailleurs, il indique que le sevrage représente «un ensemble de symptômes caractéristiques provoqués par l'arrêt brusque de consommation d'un psychotrope» (Tremblay 1988 : 243). Si la dépendance physique aux psychotropes se manifeste par des réactions d'ordre psychophysiologique lors du sevrage, la dépendance psychologique, elle, représente un processus où la personne recourt à la consommation de drogues pour se sentir bien et pour s'adapter plus facilement à la réalité.Tremblay (1988) ajoute que la dépendance psychologique est reliée à l'expérience subjective. Ainsi, la dépendance psychologique peut s'expliquer par la façon dont les femmes violentées interprètent l'effet de la drogue et envisagent la vie.

La réalité quotidienne des femmes violentées et consommatrices de drogues fait qu'elles ignorent souvent le potentiel de dépendance lié à de nombreux médicaments prescrits sur ordonnance.Ainsi, il peut arriver, suite à l'arrêt de la médication, qu'elles ne remarquent pas les symptômes d'un sevrage subi (FRT 1995). D'autres facteurs individuels peuvent aussi influencer les effets des drogues chez une femme, notamment la corpulence, l'âge, le sexe, l'alimentation, l'état de santé, l'humeur et le contexte (FRT 1995 :18). Les nombreuses caractéristiques pouvant influencer les effets des drogues font que chaque consommation peut engendrer des effets différents chez chaque femme. Les études sur le sujet démontrent que certains facteurs relatifs aux types de drogues consommées sont en relation avec les conséquences des drogues sur la santé des femmes. Ainsi, il importe de présenter quelques données quant aux types de drogues consommées pour mieux définir l'usage abusif des femmes violentées. 


\section{Les femmes et la consommation de drogues licites}

Selon certains écrits, les hommes consomment des stimulants tels que l'alcool pour gérer leur stress, tandis que les femmes consomment plutôt des psychotropes pour gérer leur état de santé. (FRT 1995). D'autres études soutiennent que les femmes sont davantage polytoxicomanes que les hommes. En effet, Nadeau et al. (1984) démontrent que les femmes alcooliques commencent à boire à un âge plus avancé que les hommes; qu'elles développent des problèmes reliés à l'alcool plus tardivement, soit vers la trentaine; qu'elles boivent le plus souvent seules, à la maison; qu'elles sont plus susceptibles d'aller chercher de l'aide. Finalement, elles concluent qu'en Amérique du Nord, une bonne proportion des femmes admises en traitement pour alcoolisme fait à la fois usage de l'alcool et d'autres psychotropes (Nadeau et al.1984).

Un sondage effectué auprès de 13000 Canadiennes et Canadiens au sujet de la consommation d'alcool et de drogues révèle que les femmes plus que les hommes, consomment des médicaments sur ordonnance tels que des tranquillisants, des antidépresseurs, des somniferes et des analgésiques narcotiques ou non-narcotiques, bien qu'il soit difficile d'estimer l'ampleur de la consommation abusive des femmes (Adlaf 1993). Ces résultats concordent avec d'autres écrits démontrant aussi la surreprésentation de la population féminine parmi les consommatrices de psychotropes (Cooperstock et Parnell 1982; Guyon 1996). Plus précisément, il semble que l'usage de tranquillisants, de somniferes et d'antidépresseurs augmente avec l'âge, particulièrement chez les femmes de soixante ans et plus et qu'il se rapporte plus fréquemment chez les personnes désavantagées sur le plan socio-économique (Guyon 1996). Dans le même sens, l'enquête effectuée par Santé Québec démontre que les personnes qui occupent un emploi ou qui poursuivent des études courent beaucoup moins le risque d'être des consommatrices de tranquillisants (Laurier et al. 1990, cité dans Landry et al. 1994). En effet, l'usage de tranquillisants au-delà de la dose prescrite et pour une durée excédant la période 
thérapeutique s'avère fréquent chez les personnes inactives (Nadeau et al. 1984). De plus, ces substances affectent le système nerveux central et engendrent un état de sédation et de passivité. Ces données suscitent des inquiétudes, étant donné qu'au Canada, les tranquillisants sont l'agent responsable du tiers à la moitié des cas de surdosage (Cooperstock et Hill 1982 cité dans Nadeau et al. 1984 : 79).

La consommation de médicaments sur ordonnance pour des «raisons de santé» peut représenter pour les femmes une norme sociale plus acceptable que la consommation excessive de drogues illicites ou d'alcool. En effet, les comportements sociaux, perçus comme déviants pour les femmes, peuvent alors les inciter à s'isoler pour consommer (Nadeau 1981). C'est pourquoi les femmes sont plus souvent perçues par les professionnels et les professionnelles de la santé comme ayant plutôt des problèmes personnels ou familiaux que des problèmes reliés à l'abus de substances (Rasmussen 1984). L'importance accordée à leur vie peut expliquer, en partie, le fait que les somniferes et les tranquillisants soient souvent prescrits aux femmes émotionnelles (Renaud cité dans Nadeau 1981). De telles substances sont prescrites pour des raisons de dépression, d'insomnie et d'anxiété alors que l'expérience de vie des femmes peut être la source de leurs émotions réprimées. L'enquête effectuée par Santé Québec révèle que la consommation de tranquillisants est reliée à une perception négative de l'état de santé, à un indice de santé globale faible, au stress, à la détresse psychologique et à un indice faible de bien-être psychologique (Laurier et al. 1990 cité dans Landry et al. 1994).

\section{Les drogues illicites : l'influence du contexte social}

En se basant sur les études portant sur l'usage abusif de drogues illicites,Abbott rapporte que les femmes comptent pour $25 \%$ des individus dépendant de drogues «de rue» telles que la marijuana, la cocaïne et l'héroïne (Abbott 1994 cité dans Bisson 1998).Alors qu'il semble que l'abus de cannabis soit plus représentatif parmi 
les femmes célibataires, séparées ou divorcées (Canada 1990), certains auteures et certains auteurs précisent que la marijuana est la drogue la plus populaire auprès de la population féminine (Yaffe, Jenson et Howard 1995 cité dans Bisson 1998). Les données portant sur la consommation de drogues illicites doivent cependant être interprétées avec précaution, étant donné qu'elles représentent un comportement illégal et que le facteur de désirabilité peut influencer les réponses (Landry et al. 1994).

Certaines études discutent des risques de contagion du VIH (virus d'immunodéficience humaine) propres aux drogues consommées par injection intraveineuse pour les femmes (Stevens et al.1998; Leonard et al.1998; Tortu et al.1998). Il en ressort que si les femmes utilisant la seringue s'injectent dans la limite de leur relation intime (Leonard et al. 1998 : 5), ce qui aurait pour effet de réduire le facteur de risque, elles demeurent néanmoins à risque de contagion parce que leur partenaire s'injecte généralement à l'intérieur d'un réseau clos d'amis et partage par la suite la seringue infectée avec sa conjointe (Friedman et al.1992; Leonard et al. 1998). Ainsi, l'utilisation de la seringue peut représenter un risque de contamination pour les femmes, comme d'ailleurs les habitudes de consommation du conjoint. Reconnaître l'influence du contexte, du «contrôle» et du «pouvoir»subis par les femmes violentées et toxicomanes permet de mettre en perspective l'ampleur du phénomène et de voir ses effets sur la vie des femmes.

\section{Liens entre consommation abusive et violence}

En 1995, une étude de la Fondation de la recherche sur la toxicomanie (FRT) analyse les liens entre les substances toxiques et la violence faite aux femmes et aux enfants. Nous en retiendrons les idées principales afin de nous permettre de mieux comprendre la signification de la consommation abusive dans le contexte de vie des femmes violentées. Cette recherche de la FRT affirme, en autres, que la consommation abusive de substances psychoanaleptiques par les femmes est surtout associée à deux 
phénomènes clés, soit le soulagement de la douleur associée à la violence présente et passée ou encore, la réduction de l'anxiété et de la douleur liée au processus de dissociation.

Lorsqu'elles sont consommées pour soulager la douleur émotive, les substances jouent le rôle de médication autoadministrée. En effet, il semble que, par ce moyen, les femmes puissent plus facilement oublier les épisodes de violence qu'elles vivent et gérer les émotions qui y sont associées. Parce qu'elles entraînent des sensations de bien-être, de sécurité, de confiance en soi et de distance face à la situation, les substances psychoanaleptiques font que la consommation représente un mécanisme de défense pour les femmes. La réduction des sentiments d'angoisse contribue aussi à la perception idéaliste d'une maitrise de la situation. Bien que la consommation soulage la douleur et refoule les souvenirs, il se peut que la situation de violence vécue par les femmes représente toujours un danger actuel. Dans ces circonstances, la consommation peut donc leur nuire à différents niveaux. En effet, si la violence persiste, ses effets peuvent difficilement être enrayés sous l'effet de psychotropes.

La dissociation est un phénomène associé aux situations représentant une violence sexuelle et physique extrême pour les femmes (FRT 1995). Il s'agit plus précisément d'un mécanisme de défense où les événements traumatisants sont refoulés de la conscience et qui permet à la personne de se détacher de la situation traumatisante. Ce mécanisme de protection personnelle empêche de sombrer dans l'angoisse. Malgré les souvenirs refoulés, les situations traumatisantes peuvent cependant refaire surface et engendrer des sentiments tels que l'anxiété et la colère. La consommation de substances associée à la dissociation atténue les malaises et réduit l'anxiété.

Bien que la consommation de substances constitue un mécanisme de survie pour contrer les effets de la violence et des émotions qui y sont rattachés, elle contribue par de nombreuses façons à maintenir les femmes dans une situation d'impuissance et à entretenir des dépendances physiques et psychologiques ayant des conséquences sérieuses pour leur santé physique et mentale. L'homme violent se sert souvent de la consommation des femmes 
pour justifier le comportement violent ou encore pour contrôler la consommation de substances de sa conjointe.

\section{Une analyse féministe de la toxicomanie féminine}

Alors que certaines approches traitent la toxicomanie féminine au niveau des comportements ou des conflits individuels et interpersonnels, ce qui peut contribuer à l'entretien d'attitudes négatives face à la consommation chez les femmes, l'analyse féministe encourage l'exploration de facteurs sociaux dans le processus de dépendance à l'alcool et aux drogues. Les écrits considèrent plusieurs facteurs qui influencent leurs conditions de vie, comme la monoparentalité (Finkelstein 1994), la violence conjugale (Gustavsson et Rycraft 1994), la situation financière précaire (Azzi-Lessing et Olsen 1996), l'isolement social (Luthar et Walsh 1995) et les abus sexuels et physiques (Finkelstein 1994). Bien qu'en situation de crise, la consommation de substances aide les femmes à surmonter des processus émotionnels difficiles, celle-ci peut conduire par ailleurs à l'acceptation passive de leurs conditions de vie plutôt qu'à la remise en question des structures qui les maintiennent dans de telles situations sociales.

Selon une perspective féministe, l'abus de substances s'explique par les structures sociales qui prédisposent les femmes à développer un tel comportement. Ce n'est pas un hasard que les femmes se retrouvent en situation d'impuissance plus souvent que les hommes. Selon Prud'homme (1994), la socialisation prépare les femmes à tolérer et à porter la responsabilité des agressions subies avant même d'en avoir été la victime. Dès l'enfance, la socialisation apprend aux filles à se centrer sur les besoins des autres au détriment de leurs propres besoins afin d'accéder à l'approbation des autres. Elles apprennent à être soumises aux hommes tout en étant douces, compréhensives, raisonnables, prudentes et responsables des comportements de ceux-ci. En plus de guider les filles à se conformer, la socialisation sert à maintenir un certain ordre social. Enfin, «un coup d'œil rapide sur la situation des femmes nous 
montre que, globalement et massivement, notre culture impose aux femmes des modèles de dépendance, de soumission et de service (ou servitude), auxquels elles ne peuvent répondre qu'au prix de leur propre santé» (Guyon et al. 1981 : 144). Subissant cette oppression sociale, les femmes doivent payer un prix énorme, autant au niveau de leur santé physique et psychologique qu'au niveau de leur qualité de vie.

\section{Réponse à la recherche d'aide des femmes}

Ayant identifié, lors d'un stage de formation pratique, que les femmes violentées éprouvent des sentiments de honte à l'idée de rechercher une aide auprès des organismes, nous avons cru bon d'explorer les réponses d'aide qui ont un impact pour les femmes en situation de violence conjugale et consommant des drogues. Ces deux problématiques mises en relation ont été retenues parce qu'elles sont représentatives du vécu des femmes et aussi, parce que les récents ateliers de formation, portant sur «Le Lien» (FRT 1995), ont soulevé beaucoup d'intérêt dans la région de Stormont, Dundas et Glengarry.

Nous avons donc invité les représentantes et les représentants des organismes de cette région desservant une population féminine et traitant de la problématique de violence conjugale et de la consommation de substances toxiques, à participer à une entrevue individuelle d'une durée d'une heure et demie à deux heures. Pour respecter les règles de confidentialité et l'anonymat des répondants, nous rapportons leurs propos sans qu'il soit possible de les identifier.

Cette démarche nous a semblé pertinente puisqu'elle permet d'examiner le fonctionnement social et les stratégies de maintien de l'ordre (Geller 1991; Regroupement provincial des maisons d'hébergement 1994). En effet, bien que la société interdise les actes de violence criminels et promeuve les valeurs du respect d'autrui et de la sécurité sociale, un mécanisme plus subtil de contrôle social s'instaure et reconduit les rapports de force entre 
les sexes et à ce titre, contribue à perpétuer la violence conjugale. Ainsi, les structures sociales visant parfois le contrôle de certaines populations (Geller 1991) jouent un rôle dans le maintien de la violence conjugale (MacLeod 1980).

L'exploration de cette dimension par les entrevues nous semble d'autant plus importante que les écrits sur le sujet montrent que la recherche d'aide des femmes violentées est un processus interactif avec l'environnement (Bilodeau 1987). Les réponses d'aide à leur demande initiale peuvent influencer les femmes dans la suite à donner à leurs initiatives (Dobash et Dobash 1985) et dans leurs réactions face à la violence subie (Bilodeau 1987). L'exploration des réponses d'aide permet d'identifier comment les services répondent effectivement aux besoins des femmes violentées lorsqu'elles recherchent de l'aide. De plus, une analyse féministe du processus interactif offrira une explication théorique de la situation vécue par les femmes.

Parmi les réponses de l'environnement ou les ressources offertes pour combler la recherche d'aide des femmes violentées et consommatrices de substances toxiques, il existe des groupes de soutien où elles peuvent partager leur vécu. Ces groupes, comme les Alcooliques Anonymes, facilitent le cheminement des femmes vers l'autonomie et l'indépendance. Selon les propos des responsables d'organismes, ces groupes misent sur la valorisation personnelle et sur le soutien continu. En plus de créer des liens sociaux, le partage du vécu personnel favorise la création des liens d'entraide au sein du groupe, encourageant ainsi le développement de nouveaux réseaux. Cela dit, la littérature sur le sujet démontre que les groupes de soutien ayant une approche traditionnelle comme les Alcooliques Anonymes, ne sont pas toujours adaptés aux besoins spécifiques des femmes (Kaplan 1991).

Par ailleurs, plusieurs agences associent le bien-être de la femme au bien-être de leur famille. Quelques-unes considèrent que les maisons d'hébergement sont des ressources au service des familles tandis que d'autres privilégient l'intervention familiale dans le cas de violence conjugale. Il en ressort qu'il pourrait y avoir des contradictions entre le fait de respecter le choix des femmes 
comme individu et celui de les encourager à protéger leur famille. Ces approches soutiennent l'hypothèse que les comportements des membres de la famille se complètent et se renforcent (RinfretRaynor et Cantin 1994; MacLeod 1980; MacLeod 1990). Cependant, maintenir l'unité familiale à tout prix tend à ignorer le déséquilibre du pouvoir dans la dynamique du couple alors que les «droits, la sécurité et les expériences des femmes et des enfants sont niés, malgré la violence et la destruction» (MacLeod $1990: 11)$.

\section{Quelques pistes à envisager}

Entre autres, les représentantes et les représentants de services suggèrent une plus grande reconnaissance du lien entre l'abus de substances et la violence conjugale. Pour certaines et certains, cette reconnaissance passe par l'adoption de politiques formelles dans la philosophie opérationnelle du service, alors que, pour d'autres, il faut développer des ressources adéquates et spécifiques pour les femmes, voire même offrir des sessions de formation par des experts et destinées au personnel, aux intervenantes et aux intervenants.

De même, le dépistage et l'évaluation approfondie des besoins individuels peuvent faciliter le processus d'aide et les démarches personnelles entreprises par la femme. Pour une évaluation juste du besoin, la connaissance acquise des problématiques est un atout. Des liens bien établis et entretenus entre les services et la collaboration entre les agences facilitent un tel processus. Pour ce faire, on suggère l'élimination d'obstacles bureaucratiques pour développer des protocoles d'échanges de services.

Certaines dirigeantes et certains dirigeants d'organismes estiment nécessaire d'accorder une importance primordiale à la socialisation et aux messages transmis aux enfants par la société. Selon certains, l'enseignement de valeurs positives peut contribuer au changement d'attitudes négatives envers les femmes dans la société et cette sensibilisation doit commencer avec la socialisation 
primaire des enfants. Des campagnes d'éducation publique et d'éducation précoce dans les écoles sont envisagées. Plus précisément, on note l'importance d'aborder avec les jeunes des thèmes tels les comportements violents non acceptables, la responsabilité personnelle, les conséquences à assumer de ses gestes et les bonnes façons d'établir une relation intime. La prévention doit aussi toucher des thèmes tels le respect de soi et de l'autre, les signes de pouvoir et de contrôle, la confiance personnelle, les limites et la confrontation. Dans tous les cas, le message doit être clair : la violence est inacceptable, et ceci, afin de briser le silence et de démystifier le caractère secret entourant la violence faite aux femmes.

Par ailleurs, les dirigeantes et les dirigeants d'organismes mentionnent l'importance d'évaluer périodiquement ce qui fonctionne dans leur organisme. Le fait d'identifier le rôle de l'agence dans la gestion d'un réseau plus large peut contribuer à considérer les liens entre la violence et l'abus de substances pour les femmes. En parallèle, les politiques de l'organisme doivent évoluer en lien avec le développement de nouveaux moyens d'offrir les services.

Outre ces propos, on note qu'avec les moyens actuels, des liens entre les organismes sont déjà présents et actifs, ce qui pourrait faciliter la mise en place de protocoles d'échanges de services. La réalité des milieux ruraux est cependant peu prise en compte sauf lorsque l'on évoque brièvement la nécessité de créer des satellites regroupant quelques organismes spécifiques.

\section{Conclusion}

Malgré la gravité des actes de violence conjugale dont les femmes sont encore aujourd'hui l'objet, elles craignent toujours de faire une demande d'aide suite à une telle expérience. Craignentelles une réponse d'aide défavorable ou la présence d'attitudes négatives? 
Le caractère secret entourant le phénomène de la violence conjugale constitue une entrave à la recherche d'aide des femmes. Étant donné les effets désastreux de la violence sur leur santé, le prix à payer pour les femmes est énorme. Des attitudes négatives entretenues par la société ou même par les membres du réseau familial des femmes minimisent la situation qu'elles vivent. Ces attitudes contribuent à «victimiser» et à isoler les femmes, alors qu'elles ont besoin d'un soutien émotionnel essentiel à leur survie. Dans leur demande d'aide, il faut reconnaitre leur courage de même que leur projet personnel pour se réapproprier un pouvoir sur leur vie.

La violence conjugale ne peut pas s'expliquer par les caractéristiques personnelles des femmes puisque celles-ci subissent les agressions dirigées contre elles. La violence représente une dynamique de contrôle par laquelle la société assure le pouvoir des hommes auprès des femmes.

Pour plusieurs femmes violentées, fuir cette oppression sociale devient un recours pour sauvegarder leur bien-être. La consommation de substances toxiques devient alors trop souvent un moyen de survie, masquant des sentiments de culpabilité et d'impuissance, fortement refoulés. Une dépendance physique et psychologique à certains psychotropes les amène à perdre de vue les effets de la violence sur leur vie.

Les résultats de notre étude démontrent plusieurs obstacles que peuvent rencontrer les femmes dans leur recherche d'aide, notamment le manque de reconnaissance du lien entre toxicomanie et violence lors du dépistage. En effet, on constate que les professionnels sont parfois mal à l'aise d'initier une conversation avec les femmes sur ce thème, tandis que les femmes ne révèlent pas facilement cette situation complexe par crainte du jugement des autres.

Bien que le vécu des femmes soit généralement accueilli sans jugement lors de demande d'aide, on peut noter que certains discours sociaux peuvent influencer le fonctionnement des organismes, comme le maintien de valeurs patriarcales à travers la préservation de l'unité familiale tend à reconduire le phénomène 
de la violence faite aux femmes. Si tel est le cas, il se peut que la recherche d'aide des femmes se traduise en une expérience "victimisante», surtout si la femme se sent socialement inadéquate en raison de sa consommation abusive ou de son vécu de violence.

Parmi les services reconnus comme étant les plus positifs pour les femmes, les groupes d'information, d'entraide et de soutien les aident à retrouver un milieu sécurisant pour discuter de leur vécu, tout en créant un réseau de partage, ce qui brise l'isolement et laisse d'autres portes ouvertes pour un soutien continu.

Par ailleurs, comme les services n'abordent pas simultanément la coexistence des phénomènes de violence et d'abus de substance, un manque important se fait donc sentir pour les femmes en recherche d'aide. Une évaluation juste de la problématique faciliterait le développement d'une intervention juste qui pourrait avoir des effets bénéfiques pour les femmes.

Il est important de constater que, selon les solutions proposées, les dirigeantes et dirigeants d'organismes font preuve de motivation pour organiser de nouveaux services et évoquent des pistes pour contribuer aux changements d'attitudes négatives dans la société.

\section{Bibliographie}

ADLAF,E.M. (1993). «Alcohol and Other Drug Use», dans T. Stephens et D.F. Graham (sous la dir. de), Canada's Health Promotion Survey 1990 :Technical Report, Ottawa, Santé Canada, 103-123.

AZZI-LESSING, L. et L.J. OLSEN (1996). «Substance Abuse-Affected Families in the Child Welfare System : New Challenges, New Alliances», Social Work, vol. 41, no 1, 15-23.

BIENER, L. (1987). "Gender Differences in the Use of Substances for Coping», dans R.C. Barnett, L. Biener et G.K. Barch (sous la dir, de), Gender and Stress, New York, Free Press, 330-349.

BILODEAU, Angèle (1987). Recherche d'aide des femmes, Québec, Les Publications du Québec.

BISSON, Geneviève (1998). J'ai un problème... de consommation. L'approche féministe pour les femmes toxicomanes, Mémoire de maitrise, École de Service social, Université d'Ottawa.

CANTIN, Solange et Maryse RINFRET-RAYNOR (1994). «Quitter le conjoint violent comme stratégie de résolution du problème», dans Maryse Rinfret-Raynor et Solange Cantin (sous la dir. de), Violence conjugale. Recherches sur la violence faite aux femmes en milieu conjugal, Boucherville, Gaëtan Morin éditeur, 265-280.

COOPERSTOCK, Ruth et Penny PARNELL (1982). «Research on Psychotropic Drug Use : A Review of Findings and Methods», Social Science and Medicine, vol. 83, 1179-1196. 
DOBASH, R. et E. DOBASH (1985). «The Contact Between Battered Women and Social and Medical Agencies", dans J. Pahl (sous la dir.de), Private Violence and Public Policy, London, Routledge and K. Paul, 142-165.

FINKELSTEIN, N. (1994). «Treatment Issues for Alcohol — and Drug — Dependent Pregnant and Parenting Women", Health and Social Work, vol.19, no1, 7-15.

FONDATION DE LA RECHERCHE SUR LA TOXICOMANIE (FRT) (1995). Le Lien, Septembre, Toronto, FRT.

FRIEDMAN, S. R, M. SUFIAN, R. CURTIS,A. NEAIGUS et D. DESJARLAIS (1992). «Organizing Drug Users Against AIDS», dans Joan Huber et Beth E. Schneider (sous la dir. de), The Social Context of Aids, Newsbury Park, Sage Publications.

GALLEY, Diane et Annette WERK (1994). «Écouter ces femmes qui quittent un conjoint violent», dans Maryse Rinfret-Raynor et Solange Cantin (sous la dir. de), Violence conjugale. Recherches sur la violence faite aux femmes en milieu conjugal, Boucherville, Gaëtan Morin éditeur, 251-262.

GELLER, Gloria (1991). Justice for Women Victims and Survivors of Abuse, Regina, Sask. Faculty of Social Work, University of Regina.

GROEMEVELD, Judith et Martin SHAIN (1989). Drug Use Among Victims of Physical and Sexual Abuse, Toronto, Addiction Research Foundation.

GUSTAVSSON, N.S, et J. R. RYNCRAFT (1994). "Chemically Dependent Mothers and Their Children", Journal of Social Service Research, vol. 20, nos 1-2, 55-71.

GUYON, Louise (1996). «Les habitudes qui agissent sur la santé», dans C. Robitaille, M. Clarkson et C. Lavallée (sous la dir. de), Derrière les apparences : santé et conditions de vie des femmes, Québec, ministère de la Santé et des Services sociaux.

GUYON, L., R. SIMARD et L. NADEAU (1981). Va te faire soigner, t'es malade!, Québec, Stanké.

HEALTH AND WELFARE CANADA (1990). National Alcohol and Other Drugs Survey (1989): Highlights Report, Ottawa, Health and Welfare Canada.

JONES, Ann et Susan SCHECHTER (1992). Quand l'amour ne va plus. Échapper à l'emprise d'un conjoint manipulateur, Québec, Éditions Le jour.

KAPLAN, Sarah (1991). «Child Abuse and Alcoholism in Women:A Feminist Approach to Treatment», Canadian Woman Studies vol. 12, no 1,67-70.

LANDRY, M., P. LAMARCHE, J. BOILARD et L. NADEAU (1994). «Alcoolisme et autres toxicomanies», dans Fernand Dumont, Simon Langlois et Yves Martin (sous la dir. de), Traité des problèmes sociaux, Québec, Institut québécois de recherche sur la culture, 179-195.

LEMIEUX, Denise (1994). «La violence conjugale», dans Fernand Dumont, Simon Langlois et Yves Martin (sous la dir. de), Traité des problèmes sociaux, Québec, Institut québécois de recherche sur la culture, 337-361.

LEONARD, Lynne, Hotz STEPHEN et Janice HANSEN (1998). Injecting Gender : A Gendered Analysis of Injection Drug Users' HIV-Related Sexual and Injection Practices, Québec, Seventh Annual Canadian Conference on HIV/AIDS Research.

LIMANDRI, B.J. (1985). Help-Seeking Patterns of Abused Women: Self-Esteem, Role Conflict and Social Support as Influencing Factors, thèse de doctorat en Nursing, San Francisco, University of California.

LUTHAR, S.S. et K.G. WALSH (1995). «Treatment Needs of Drug-Addicted Mothers- Integrated Parenting Psychotherapy Interventions", Journal of Substance Abuse Treatment, vol. 12, no 5, 341348. 
MACLEOD, Linda (1990). Le counselling: un outil de changement, Ottawa, Santé et Bien-être social Canada.

MACLEOD, Linda (1980). Wife Battering in Canada : The Vicious Circle, Ottawa, Canadian Advisory Council on the Status of Women.

NADEAU, L., C. MER CIER et L. BOURGEOIS (1984). Les femmes et l'alcool en Amérique du Nord et au Québec, Université du Québec.

PRAIRIE, Joanne (1984). Violence conjugale : processus d'arrêt, thèse de maitrise en criminologie, Montréal, Université de Montréal.

PRUD'HOMME, Diane (1994). La victimisation des femmes : quand ça commence et où ça finit, Montréal, le Regroupement provincial des maisons d'hébergement et de transition pour femmes victimes de violence conjugale.

RASMUSSEN, Linda (1984). «Why Women Drink», Healthsharing, fall, 9-11.

RATNER, P. (1993). «The Incidence of Wife Abuse and Mental Health Status in Abused Wives in Edmonton, Alberta", Canadian Journal of Public Health, vol. 84, no 4, 246-250.

REGROUPEMENT PROVINCIAL DES MAISONS D'HÉBERGEMENT ET DE TRANSITION POUR FEMMES VICTIMES DE VIOLENCE CONJUGALE (1994). Un grain de sable dans l'engrenage : pistes de solution pour contrer la violence conjugale, Montréal, RPMHTFVVC.

RINFRET-RAYNOR, Maryse et Solange CANTIN (1994). «Violence conjugale rapportée par des femmes s'adressant aux services sociaux", dans Maryse Rinfret-Raynor et Solange Cantin (sous la dir. de), Violence conjugale. Recherches sur la violence faite aux femmes en milieu conjugal, Boucherville, Gaëtan Morin éditeur, 3-22.

STATISTIQUE CANADA (1994). «Résultats d'une enquête nationale sur l'agression contre la conjointe», Statistique Canada, no 85-002 au catalogue, vol.14, no 9,11.

STEVENS, S., A. ESTRADA et B. ESTRADA (1998). «HIV Sex and Drug Risk Behavior and Behavior Change in a National Sample of Injection Drug and Crack Cocaine Using Women", Women and Health, vol. 27, nos1-2, 25-48.

TORTU, S., S. J. STEVENS et S. COYLE (1998). «Women Drug Users and HIV Prevention : Overview of Findings and Research Needs», Women and Health, vol. 27, nos 1-2, 19-23.

TREMBLAY, Roch (1988). «Les toxicomanes», dans F. Lalonde et al. (sous la dir. de), Psychiatrie clinique : approche bio-psycho-sociale, Boucherville, Gaëtan Morin éditeur, 238-264.

\section{Note}

1. Octroyée depuis 1998 à une étudiante de l'École de service social, Université d'Ottawa, la bourse du Regroupement des intervenantes et des intervenants contre les agressions sexuelles (RIFAS) vise à souligner l'excellence d'un mémoire de maitrise portant sur la violence. Le mémoire de Mme Rivette a été dirigé par la professeure Cécile Coderre. 